\title{
Adsorption of DOPC vesicles on hydrophobic substrates in the presence of electrolytes: A QCM and reflectometry study
}

\author{
G EDWARD GNANA JOTHI, S KAMATCHI and A DHATHATHREYAN* \\ Chemical Laboratory, Central Leather Research Institute (CSIR), Adyar, Chennai 600020 \\ e-mail: aruna@clri.res.in
}

MS received 19 August 2009; revised 23 February 2010; accepted 9 April 2010

\begin{abstract}
The adsorption of lipid Dioleoylphosphatidylcholine (DOPC) vesicles on a hydrophobic substrate has been investigated in aqueous buffer solution by means of the quartz crystal microbalance (QCM) and reflectometry. DOPC vesicles were prepared by the injection method on a hydrophobic substrate using 1-octadecanethiol (ODT) self-assembled on a gold-coated AT-cut quartz. The reflection spectrometry measurements of the adsorbed vesicles showed nearly monolayer formation in few cases, while in most other experiments, the frequency changes measured suggested multilayer formation assuming the usual Sauerbrey equation to hold in the present system as well. Presence of $\mathrm{NaCl}, \mathrm{KCl}, \mathrm{Na}_{2} \mathrm{SO}_{4}$ and ethanol in the aqueous phase during the formation of vesicles suggest that the multilayer formation can be hastened in some cases. Atomic force microscopic study corroborate the thicknesses that range between 8 and $20 \mathrm{~nm}$ for high concentration of electrolytes or ethanol suggesting coalescence of vesicles leading to several bilayers possibly stacked over each other.
\end{abstract}

Keywords. DOPC vesicles; supported multi-bilayers; adsorption; rupture; QCM; electrolytes.

\section{Introduction}

A variety of lipids under special conditions are known to form thermodynamically stable bilayers on solid substrates that are widely used as models to understand cell membranes. When these vesicles contact solid substrates, supported lipid bilayers (SLBs) are formed and these allow the study of membrane constituents in a controlled environment. ${ }^{1-3}$

The simple geometry and the robustness given by a solid surface allow that SLBs can be studied using a variety of optical, spectroscopic, microscopic, and other surface sensitive techniques. Modifying solid surfaces using SLBs is important for studying biological reactions and in the development of new devices and biosensors. ${ }^{4}$ Atomic force microscopy (AFM), fluorescence microscopy based methods, quartz crystal microbalance and surface plasmon resonance methods have been used to analyse structures of SLBs. ${ }^{5-10}$ Leermakers et al have recently published an elegant work on entropic stabilization and equilibrium sizes of lipid vesicles. ${ }^{11}$

*For correspondence
Studies of membrane protein interactions often require use of SLBs with proteins on solid supports. These systems have been analysed using classical methods such as electron diffraction and also recently introduced techniques for membrane study at (sub)- nanometer resolution, like surface plasmon resonance $(\mathrm{SPR})^{12}$ total internal reflection fluorescence (TIRF), ${ }^{13}$ atomic force microscopy (AFM) ${ }^{14}$ quartz crystal microbalance with dissipation monitoring (QCM-D) $)^{15}$ and fluorescence correlation spectroscopy (FCS). ${ }^{16}$

During formation of vesicles, lipids usually bind considerable amounts of water at their head groups, often establishing a liquid-crystalline lamellar arrangement. This phase consists of an alternating stack of planar, undisrupted bilayers and intervening layers of solvent. This lipid hydration is known to affect not only the molecular interactions in the head group region, but also the organization and motions of the acyl chains comprising the hydrocarbon core. ${ }^{17}$ Thus an understanding of the role of hydration in forming SLBs and the different structural arrangements that these layers can take up is of interest.

In the present study, hydrodynamically coupled solvent, film mass and the work of adhesion of SLBs of Dioleoylphosphatidylcholine (DOPC) vesi- 
cles from quartz crystal microbalance (QCM) have been used for the first time to understand the adhesion of these SLBs on hydrophobic gold surface. This technique allows real-time monitoring of surface mass, changes in contact angles of the fluid drops and thus of adsorption and transition kinetics of fluids on solid surfaces.

The influence of electrolytes $\left(\mathrm{NaCl}, \mathrm{Na}_{2} \mathrm{SO}_{4}\right.$ and $\mathrm{KCl})$ and ethanol (1\% and 5\%) on the hydration of the vesicles has been analysed and their role in spreading and organization of the bilayers studied.

\section{Materials and methods}

\subsection{Materials}

The synthetic phospholipid, Dioleoylphosphatidylcholine (DOPC) was purchased from Larodan Chemicals (99.9\% pure), Sweden and stored below $-5^{\circ} \mathrm{C}$ before use. 1-Octadecanethiol (ODT) (purity 99.9\%) was purchased from Aldrich and used as received. All other chemicals were reagent grade unless specified otherwise, and deionised water, of resistivity around 18.0 M $\Omega$ (Millipore-Milli-Q system) was used throughout. Electrolytes $\mathrm{NaCl}$, $\mathrm{Na}_{2} \mathrm{SO}_{4}, \mathrm{KCl}$ of ANALAR grade and HPLC grade ethanol were obtained from Merck, India.

\subsection{Vesicle preparation}

The lipid vesicle solution of DOPC was prepared by the injection method. ${ }^{18,19}$ Initially, $20 \mathrm{mg}$ of lipid dissolved in $1 \mathrm{~mL}$ of chloroform was evaporated under laboratory atmosphere. The residue was re-dissolved in $500 \mu \mathrm{L}$ of isopropyl alcohol and then injected, using a syringe, into a stock buffer solution (20 mL at $\mathrm{pH} 7.4$ and $2 \mathrm{mM}$ EDTA) at $45^{\circ} \mathrm{C}$ under vigorous stirring. After $20 \mathrm{~min}$, the solution was cooled to room temperature. Any visible lump was removed by centrifuging at $3000 \mathrm{rpm}$. The size of the remaining vesicles was determined to be about $100 \mathrm{~nm}$ by means of the dynamic light scattering (Brookhaven Instruments Co.), suggesting the formation of multilamellar vesicles (MLVs). The MLV solution once prepared was used throughout the whole experiment; when recycling was needed, the vesicle solution was briefly sonicated for $2 \mathrm{~min}$ before use. The prepared vesicles were refrigerated at $8^{\circ} \mathrm{C}$ and were stable for at least 2 to 3 weeks.

For preparing the DOPC vesicles with the electrolytes the buffer with the defined amount of electro- lytes (5 to $20 \mathrm{mM}$ of $\mathrm{NaCl}, 10 \mathrm{mM}$ of $\mathrm{Na}_{2} \mathrm{SO}_{4}$, $10 \mathrm{mM}$ of $\mathrm{KCl}$ ) and $1 \%$ and $5 \%$ ethanol were used. The gold surfaces (on quartz used in QCM and also for reflection measurements) were boiled in methanol or treated with Hellmanex II (Hellma, Mullheim, Germany) and sonicated. Before use the surfaces were rinsed with deionized Milli-Q water.

\subsection{QCM measurements}

In case of QCM measurements, the Au-coated crystals were left in contact with $1 \mathrm{mM}$ decanethiol (ODT) solution in ethanol for $2 \mathrm{~h}$ for the gold surface to become fully hydrophobic by the self-assembly of ODT on gold as thiolate. The modified gold surface was subsequently washed with excess absolute ethanol to remove any physisorbed ODT molecules and then blown dry by a $\mathrm{N}_{2}$ gas jet. This substrate was used to study the formation of SLBs of DOPC vesicles with electrolytes and ethanol respectively. In order to check the SAM-coated surface for hydrophobicity, contact angles of sessile drops of water were measured with a custom built instrument before each measurement. The average contact angle was around $86^{\circ}$ while that of the bare gold surface was around $58^{\circ}$.

The QCM cell was filled with $1 \mathrm{~mL}$ of phosphate buffer solution $(\mathrm{pH}=7.4)$, after connecting to the oscillator circuit, and thermostated at $20^{\circ} \mathrm{C}$. Waiting for $10 \mathrm{~min}$ for equilibrium, the buffer was exchanged with $1 \mathrm{~mL}$ of DOPC vesicle solution into the cell using a syringe, and subsequently the frequency change as a function of time was monitored. After about half an hour, the vesicle solution was removed and any excess unabsorbed vesicles removed by washing with buffer. This procedure was repeated for all the vesicles with the electrolytes and ethanol.

The QCM technique is, based on the well-known equation formulated by Sauerbrey in $1959^{20}$ which relates a decrease in the resonance frequency of a quartz crystal, $f$, to accumulation of mass, $m$, caused by the deposition of amounts of material at the crystal surface,

$$
m=C \Delta f / z,
$$

where the mass sensitivity constant is $C=-17.7 \mathrm{ng} /$ $\left(\mathrm{cm}^{2} \mathrm{~Hz}\right)$ for a $5 \mathrm{MHz}$ fundamental mode AT cut quartz crystal, and $z=1$ is the overtone number here.

Muthuselvi and Dhathathreyan have used the QCM to evaluate the coupled solvent fraction of 
amino acids, peptides and proteins ${ }^{21,22}$ from a modified method of Craig and Plunkett. ${ }^{23}$ Using this method, in the present study, the vesicles prepared in water and in $\mathrm{D}_{2} \mathrm{O}$ have been analysed and the coupled solvent fraction of water in vesicles is evaluated.

Knowing the density of the dispersions in water and in $\mathrm{D}_{2} \mathrm{O}$, one can obtain the fraction of total mass due to the bound water

$$
\text { Solvent fraction }=\left(\Delta f_{s}-\Delta f_{D}\right) /\left(\Delta f_{s}\left(1-\rho_{D} / \rho_{s}\right)\right)
$$

From the solvent fraction, mass of the film and that of the hydrodynamically bound water may be estimated using

Film mass $=\Delta f_{s} /-C(1-$ solvent fraction $)$

$$
\text { Solvent mass }=\Delta f_{s} /-C(\text { solvent fraction }) \text {. }
$$

Reflectometry technique has been used to measure the thickness (hereafter referred as optical thickness) of the SLBs formed from the vesicles spreading on the hydrophobic gold surface. A laser diode of wavelength $=635 \mathrm{~nm}$ (power $10 \mathrm{~mW}$ ) at a fixed angle of incidence is used with polarizer and the intensities of parallel (p-) and perpendicularly (s-) polarized reflected light from the gold surface is recorded. The reflected intensities are measured using photodiodes. Then the surface is covered with the vesicles and again the intensities are recorded. Here the intensity ratio $S=I_{\mathrm{p}} / I_{\mathrm{s}}$ changes upon adsorption and spreading of the vesicles. This method has been used in combination with QCM-D by Kasemo et al to analyse different supported lipid structures and their interactions with biomolecules. ${ }^{24}$

Knowing $\Delta \mathrm{R}$ which is $\Delta R=\left(S-S_{0}\right) / S_{0}$ and from the refractive indices $\mathrm{n}$ and $\mathrm{n}_{0}$ of the films (measured using Abbey refractometer), the thickness d can be evaluated from

$$
d=\Delta R / A\left(n-n_{0}\right) .
$$

$A$ is the sensitivity factor ${ }^{25}$ which is dependent on the optical set-up (angle of incidence, wavelength of the polarized light, and properties of the substrate and the surrounding solvent). Here the subscript ' 0 ' denotes bare substrate.

Contact angle of the spreading vesicles have been estimated using the method of Lin and Ward ${ }^{26}$ The contact angles were measured as a function of time parallel with the measurements of delta frequency and thickness changes. Here the assumption is that the refractive indices of the vesicles and the buffer are close to each other at the time of spreading and the frequency change associated with an area of liquid droplet in contact

$$
\begin{aligned}
\Delta f=\Delta f=\iint S(r, \theta) m(r, \theta) r \mathrm{~d} r \mathrm{~d} \theta \\
\text { for limits } 0 \text { to } 2 \pi \text { and } 0 \text { to } r_{e}
\end{aligned}
$$

\section{$S$, differential sensitivity $(\partial f / \partial m)$.}

For a Newtonian fluid $m(r, \theta)$ is described as the effective Mass contained within decay length $\delta$

$$
\delta=\left(\eta_{1} / \pi f_{0} \rho_{1}\right)^{1 / 2} .
$$

Using Sauerbrey's (1) and (6) and (7).

$$
\begin{aligned}
\Delta f= & \mathrm{C}\left[\left(3 V_{d} / \pi\right)^{2 / 3}\left(1-\cos ^{2} \theta\right)\right] / \\
& {\left[\left(2-3 \cos \theta+\cos ^{3} \theta\right)^{2 / 3}\right] . }
\end{aligned}
$$

The contact angle $\theta$ is evaluated. From this value, the work of adhesion for the vesicles interacting with the gold surface is given by

$$
W_{\mathrm{ads}}=\gamma_{\mathrm{ves}}(1+\cos \theta) \text {. }
$$

Here $\gamma_{\text {ves }}$ was obtained by using the conventional Wilhelmy balance technique and the solutions containing the vesicles was used to measure the surface tension values.

Using Young's equation and assuming a constant interfacial tension value $\gamma$ for the different interfaces, a force balance along the solid surface at the three phase contact line gives the contact angle.

$$
\gamma_{\text {ves }} \cos \theta=\gamma_{\text {gold }}-\gamma_{\text {gold } / \text { ves }}
$$

Using the correspondence between monolayer and bilayer, the surface tension $\gamma_{\text {ves }}$ was estimated and values ranged between $59 \mathrm{mN} / \mathrm{m}$ and $65 \mathrm{mN} / \mathrm{m}$ for the pure DOPC vesicles and with the additives.

\subsection{Atomic force microscopy}

A digital instrument nanoscope III AFM (Santa Barbara, CA) fitted with a $125 \mu \mathrm{m}$ scanner (J-scanner) and a tapping model liquid cell was used to image the vesicles spreading at the solid substrates. Standard $\mathrm{Si}_{3} \mathrm{~N}_{4}$ tips with a nominal force constant of $0.35 \mathrm{~N} / \mathrm{m}$ were used and the forces minimized dur- 
ing the scans. The scanning line speed has been optimized between 1 and $3 \mathrm{~Hz}$ with a $256 \times 256$ pixel number. Images have been recorded in the height mode. The height images were flattened and plane adjusted. For imaging the samples the vesicles were introduced on polished silicon wafers $(10 \times 10 \mathrm{~mm})$ placed directly in the microscope. A glass tapping mode liquid cell was used and the solution (vesicles) added using a Hamilton syringe.

\section{Results and discussion}

Normally for a vesicle to adsorb to a surface and adhere it has to experience a net attractive force. If on nearing a surface a stress is experienced by the vesicle and if this stress exceeds a certain threshold the adsorbed intact vesicle starts to rupture. Rupture is strongly promoted by the following factors: surface hydrophobicity, low electrostatic repulsion and high vesicle surface density. In this study SLBs seem to form on hydrophobic gold coated QCM crystals via spontaneous adsorption and spreading of DOPC vesicles. Representative delta frequency plots from QCM are shown in figure 1 for DOPC vesicles in water and in the presence of the electrolytes and $1 \%$ ethanol. There are several points to be addressed regarding the large frequency variation observed. First of all, as the MLV solution was injected, the frequency decrease was rather abrupt. Most research work on spreading of vesicles have reported only a frequency change of about 60 to $100 \mathrm{~Hz}$ on hydrophilic substrates. However, in our study we find the changes ranging from about 50 to $180 \mathrm{~Hz}$ for the different vesicles. Tai Hwan Ha and Kwan Kim in their work on QCM-D experiments have shown that factors like viscoelasticity, high mass loading, surface roughness, surface stress, non-uniform mass distribution, and inter-facial slippage can lead to large changes in delta frequency. ${ }^{27}$

Since the shear modulus and the viscosity of a thin film are generally not known. The adsorbed thin film is usually assumed to be very rigid.

Such an assumption may lead to the failure of the Sauerbrey equation, but there are ample examples specifically in the QCM investigations of polymer films that ordinary films can be regarded as rigid and/or changes in viscoelasticity as negligibly small during the experiment. In any QCM experiment, a mass loading not exceeding $2 \%$ of the mass of the crystal is permitted. In the present case even formation of multilayers of DOPC would still be much less than the $2 \%$ limit of the mass loading. As the DOPC vesicles are introduced into the crystal, they initially adsorb intact onto the surface, resulting in an increase in coupled mass.

On these grounds, one may assume that the unusual response of QCM was caused by the viscosity of the electrode-adjacent liquid layer being much

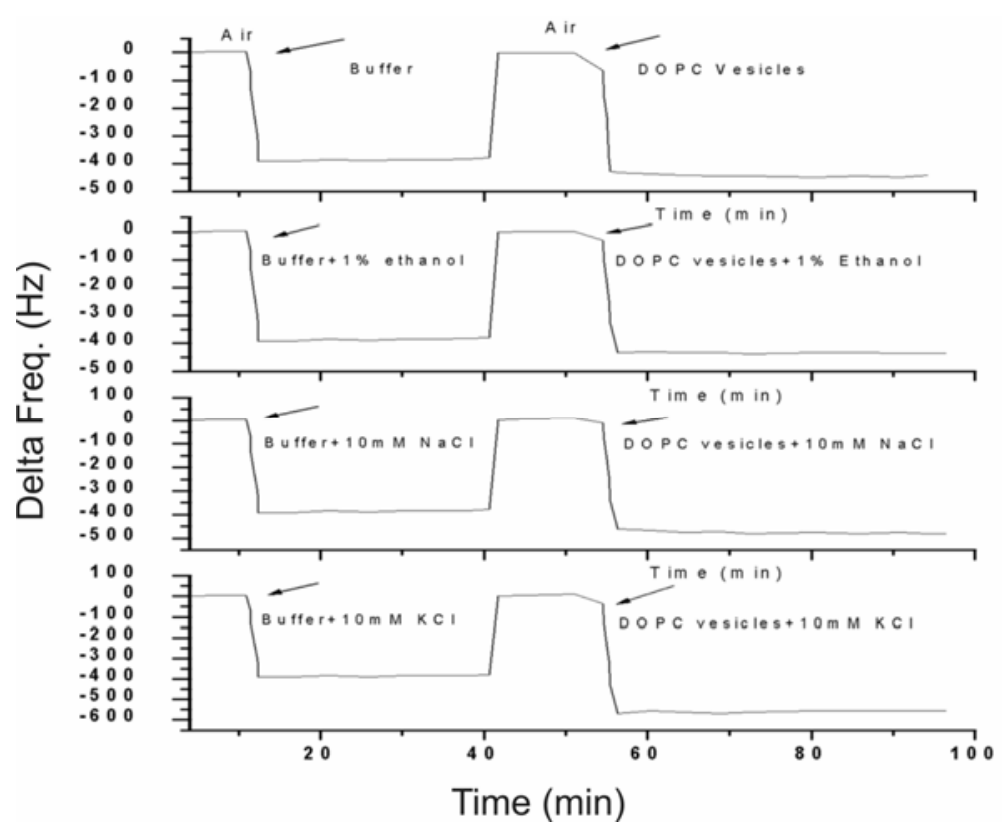

Figure 1. $\Delta f$ vs time for DOPC vesicles with water, ethanol, $\mathrm{NaCl}$ and $\mathrm{KCl}$. 

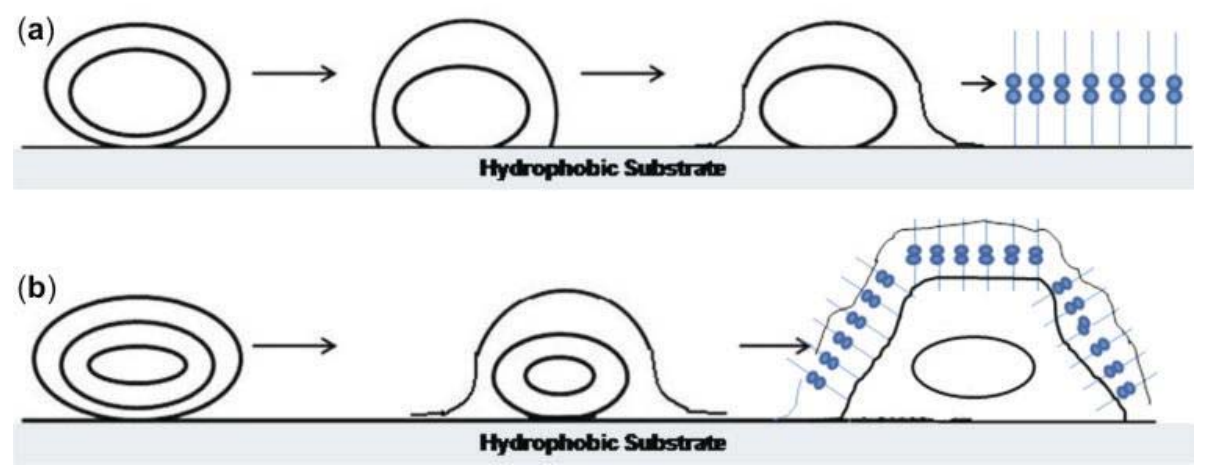

Figure 2. Spreading and rupturing of (a) regular vesicle to supported bilayers, (b) multiwalled vesicles to partially ruptured vesicles and vesicles + bilayers.
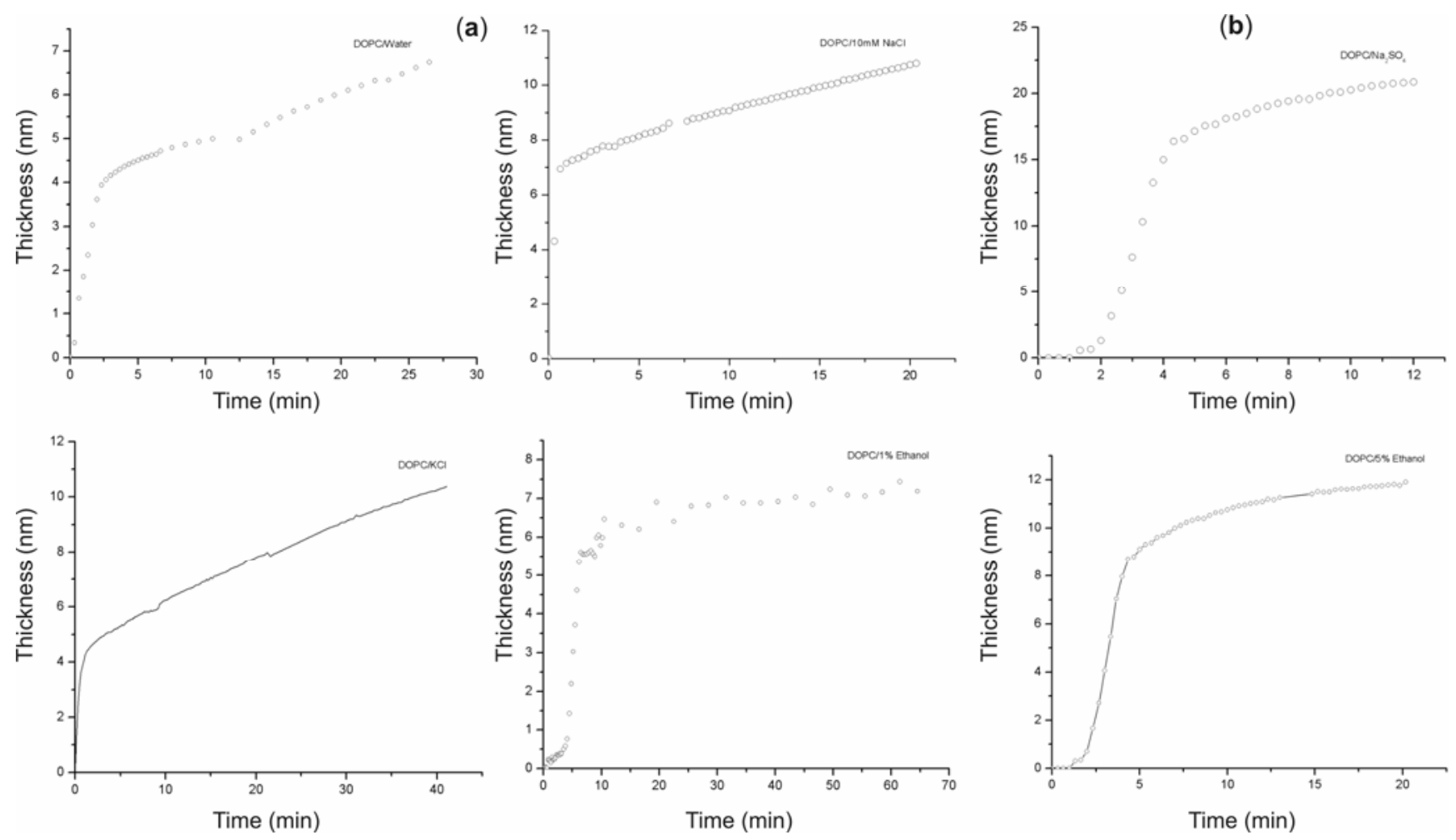

Figure 3. Thickness vs time for spreading DOPC vesicles with (a) water, $10 \mathrm{mM} \mathrm{NaCl}, \mathrm{Na}_{2} \mathrm{So}_{4}$ and $\mathrm{KCl}$, (b) $1 \%$ and $5 \%$ ethanol on gold surface.

larger than that of the bulk phase. The larger viscosity and thickness of the electrode-adjacent liquid layer are presumed due to co-existence of intact vesicles on the ODT coated surface and vesicles with partially spread bilayers stacked on top of the vesicles and thus contributing to the large delta frequency changes.

In Figure 2(a), a typical double walled vesicle on adsorption to a solid substrate starts to spread, ruptures and forms supported bilayer. In the case of a multiwalled vesicle (figure 2(b)) the adsorption of the vesicle is followed by both intact vesicles as well as partially ruptured vesicles on which bilayers could sit thereby suggesting the large change in thickness as well as the delta frequency values. The presence of small uncovered areas in the SAM coated gold surface is likely to lead to first the adhesion of the vesicles followed by the rupture due to increasing vesicular adsorption. The pathways of vesicle rupture induced by the cooperative action of neighbouring adsorbed vesicles has been described and characterized in detail by Richter and Brisson. ${ }^{28}$ 
Here the formation of SLBs at higher surface coverage confirms the influence of neighbouring vesicles in the induction of rupture of the first vesicle suggesting that the phenomenon of critical surface coverage is a necessary condition. This has been substantiated by the work of Reviakine and Brisson. ${ }^{29}$

In the corresponding reflectometry experiment there is an increase in thickness as shown in figure 3(a) and (b). As a critical surface coverage is reached, the vesicles start to fuse after rupture and form bilayers. The idea of a critical surface coverage requirement for bilayer formation has been reported by Sackmann et al. ${ }^{30}$ The reflection signal which is supposed to measure the nonhydrated adsorbed mass continues to increase monotonically as long as lipid material is adsorbing to the surface before reaching a steady state.

Jahnig et al in their work on vesicles interacting with solid surfaces have evaluated the amount of lipid adsorbed in the SLBs and verified whether complete bilayers are formed in these SLBs. ${ }^{31}$

In their method knowing the area of the substrate, surface density (from the hydrated film mass) and molecular weight of the lipid, surface particle density and area per particle have been calculated. In the present work, this method has been used to evaluate the area per particle and the area/molecule in the layers from the film mass (eqn (3)) obtained from QCM. From molecular models of DOPC it is known that a single molecule occupies an area of $0.72 \mathrm{~nm}^{2}$ in a close packed monolayer and in case of a typical bilayer to achieve similar surface density this is around $0.36 \mathrm{~nm}^{2}$. The corresponding bilayer thickness would be $6.66 \mathrm{~nm}$. Our estimates for DOPC vesicles on the hydrophobic surface forming SLBs show areas nearly that of a typical DOPC bilayer with water and $1 \%$ ethanol. In all other cases it varies from one and half bilayers to almost three bilayers ( $\left.10 \mathrm{mM} \mathrm{Na}_{2} \mathrm{SO}_{4}\right)$.

The values of thicknesses obtained from QCM (termed as acoustic thickness) and from reflectometry measurements (optic thickness) together with the work of adhesion for the SLBs are presented in table 1. From these thickness estimated it is seen that the vesicles on adsorbing to solid substrates form simple bilayers or multibilayers. Sandhya et al have recently demonstrated the usefulness of the rate of change of work of adhesion, $\Delta W$ to predict qualities of surface coatings of protein films. ${ }^{32}$ Using this parameter $\Delta W$, in the present work, the adsorption of different vesicles and their stability on the solid substrates have been analysed.

From the work of adhesion a parameter $\Delta W$ that is the difference between work of adhesion at time $t=0$ and at $t=\infty$, (expressed as $\left(W_{t}-W_{\text {eq }}\right)$ ) can be plotted as a function of time. Here $\Delta W$ is

$$
\Delta W=\gamma\left(1+\cos \theta_{\mathrm{t}}\right)-\chi\left(1+\cos \theta_{\infty}\right)
$$

or

$$
\Delta W=\gamma\left(\cos \theta_{\mathrm{t}}-\cos \theta_{\infty}\right)
$$

Therefore for $\theta$ values between 0 and $180^{\circ}$

$$
\begin{array}{lll}
\Delta W>0 & \theta_{\infty}>\theta_{\mathrm{t}} \text { Hindered adsorption } \\
\Delta W<0 & \theta_{\infty}<\theta_{\mathrm{t}} & \text { Promotion of adsorption } \\
\Delta W=0 & \theta_{\infty}=\theta_{\mathrm{t}} & \text { No adsorption. }
\end{array}
$$

There exists a condition between that of eqs (12) and (13) where difference between $\theta_{\infty}$ and $\theta_{\mathrm{t}}$ may tend towards zero. The $\theta$ values were evaluated using eqn (8).

This situation corresponds to poor adsorption due to poor spreading of the solution on the solid. Such a condition, therefore, can lead to more localized poorly spreading vesicles leading to multibilayer formation.

The acoustic and optic thickness measurements from QCM and the reflectometry agree reasonably well. At low $\mathrm{NaCl}$ concentration, the vesicles form nearly uniform SLBs. As the concentration of $\mathrm{NaCl}$ is increased or the electrolyte contains divalent cations $(\mathrm{KCl})$ or divalent anions $\left(\mathrm{Na}_{2} \mathrm{SO}_{4}\right)$ the vesicles form multibilayers. In the presence of electrolytes or high \% ethanol the vesicles organize themselves in layers that deviate from classical SLBs. If the film mass from QCM is assumed to arise from flattening of the vesicles and covering the surface, the extent of flattening is governed by the amount of water taken up by the headgroups in the vesicles. If one were to assume that DOPC vesicles do have some residual negative charge due to hydration layers near the surface as demonstrated by Egawa and Furusawa in their work on adhesion of liposomes $^{33}$ addition of electrolytes should lead to compensation of the surface charges Also depending on the monovalent or divalent cations the electrokinetic potential of the vesicles may even change from positive to negative values. Therefore from an 
Table 1. Film mass, acoustic and optical thickness and work of adhesion for DOPC vesicles.

\begin{tabular}{|c|c|c|c|c|c|c|}
\hline \multirow[b]{2}{*}{$\begin{array}{l}\text { DOPC } \\
\text { vesicles with }\end{array}$} & \multicolumn{3}{|c|}{ QCM } & Reflection method & \multirow[b]{2}{*}{$\begin{array}{l}\text { Initial rate of change } \\
\text { in } \Delta W\left(\mathrm{~mJ} / \mathrm{m}^{2}\right)\end{array}$} & \multirow[b]{2}{*}{$\begin{array}{l}\text { Final rate of change } \\
\text { in } \Delta W\left(\mathrm{~mJ} / \mathrm{m}^{2}\right)\end{array}$} \\
\hline & $\begin{array}{l}\text { Film mass } \\
\left(\mu \mathrm{g} / \mathrm{cm}^{2}\right)\end{array}$ & $\begin{array}{c}\text { Area/ } \\
\operatorname{BL}\left(\mathrm{nm}^{2}\right)\end{array}$ & $\begin{array}{c}\text { Acoustic } \\
\text { thickness }(\mathrm{nm})\end{array}$ & $\begin{array}{l}\text { Optical } \\
\text { thickness (nm) }\end{array}$ & & \\
\hline Buffer & $4 \cdot 696$ & $0 \cdot 38$ & $7 \cdot 03$ & $7 \cdot 04$ & $-3 \cdot 84$ & $0 \cdot 117$ \\
\hline $5 \mathrm{mM} \mathrm{NaCl}$ & $3 \cdot 544$ & $0 \cdot 5$ & $9 \cdot 38$ & 8.64 & $-6 \cdot 75$ & $0 \cdot 058$ \\
\hline $10 \mathrm{mM} \mathrm{NaCl}$ & $2 \cdot 855$ & 0.63 & $11 \cdot 59$ & $11 \cdot 57$ & $-20 \cdot 13$ & $0 \cdot 068$ \\
\hline $20 \mathrm{mM} \mathrm{NaCl}$ & $2 \cdot 172$ & 0.82 & $15 \cdot 23$ & $14 \cdot 57$ & $-24 \cdot 73$ & 0.059 \\
\hline $10 \mathrm{mM} \mathrm{Na}_{2} \mathrm{SO}_{4}$ & 1.452 & $1 \cdot 23$ & 22.78 & $23 \cdot 38$ & $-5 \cdot 89$ & 0.035 \\
\hline $10 \mathrm{mM} \mathrm{KCl}$ & $2 \cdot 915$ & 0.61 & $11 \cdot 35$ & $11 \cdot 54$ & $-2 \cdot 058$ & $0 \cdot 157$ \\
\hline $1 \%$ Ethanol & $4 \cdot 204$ & 0.43 & $7 \cdot 87$ & $7 \cdot 17$ & $-3 \cdot 183$ & 0.658 \\
\hline $5 \%$ Ethanol & 1.720 & 1.04 & $19 \cdot 24$ & $19 \cdot 03$ & $-92 \cdot 04$ & $-0 \cdot 101$ \\
\hline
\end{tabular}
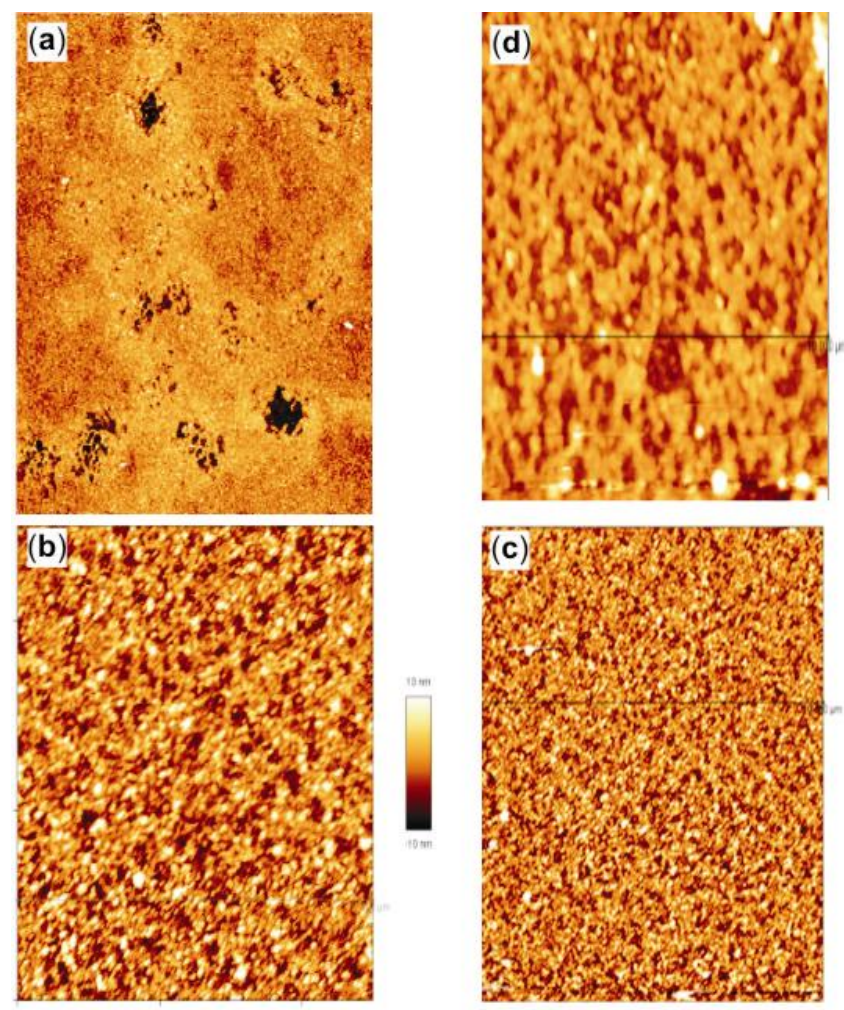

Figure 4. Atomic force micrographs of DOPC vesicles with (a) water, (b) $10 \mathrm{mM} \mathrm{NaCl}$, (c) $10 \mathrm{mM} \mathrm{KCl}$ and (d) $1 \%$ ethanol.

electrostatic point of view it can be assumed that the vesicles with high $\mathrm{NaCl}$ concentration or $\mathrm{KCl}$ or $\mathrm{Na}_{2} \mathrm{SO}_{4}$, in general promote coalescence of the vesicles that in turn may result in multibilayers.

Figures $4 a-d$ show the AFM images of DOPC vesicles with water and water $+\mathrm{NaCl}(5 \mathrm{mM}), \mathrm{NaCl}$ $(10 \mathrm{mM}), \mathrm{KCl}(10 \mathrm{mM})$ and $1 \%$ ethanol. From the images no typical spherical relief is seen except in the case of vesicles with $1 \%$ ethanol. From molecular model a typical extended bilayer of DOPC is around $6.7 \mathrm{~nm} .{ }^{1} \mathrm{H}$ NMR experiments carried out on SLBs adsorbed to glass beads have shown that there is a non-accessible volume that can be attributed to water layer between the membrane and the surface and is about $1.7 \mathrm{~nm}$ thick. ${ }^{34}$ Thus, the increased thickness of $7 \mathrm{~nm}$ seen in our study may be due to the SLBs associated with this layer of water. Table 1 shows the formation SLBs greater than the single bilayer with thickness reaching even $22.78 \mathrm{~nm}$ for vesicles with $\mathrm{Na}_{2} \mathrm{SO}_{4}$. AFM study could not be carried out on this sample because the image was too fuzzy with large amount of water between the vesicles and the surface. While the rates of adhesion and spreading may not always be exactly measurable, the initial and final values of $\Delta W$ should reflect the formation of the multibilayers. Thus in almost all the samples the initial values show that there is promotion of adhesion and specially with vesicles with $5 \%$ ethanol having the lowest $\Delta W$ value and thus the maximum propensity to coalesce faster. In case of the electrolytes as the concentration of $\mathrm{NaCl}$ increases the initial $\Delta W$ value shows a decrease and on complete spreading the $\Delta W$ is nearly zero suggesting that possibly the whole surface is now covered by the several stacked bilayers.

Zhu et al in their work on vesicles of neutral lipids have demonstrated the role of surface charge density and electrostatic interaction on the adsorption of the SLBs. ${ }^{5}$ Our results agree with their findings in that increasing concentrations of electrolytes lead to possibly unruptured vesicles sitting on single bilayers. In the present study, the interaction between an intact DOPC vesicle and the hydrophobic surface is mainly through van der Waals interaction. In presence of increasing concentrations of electrolytes, counter ions are present in the vicinity of the DOPC vesicle. As the vesicle approaches the solid substrate, the counter ions are pushed into 
smaller space between the vesicle and the solid. This eventually should lead to a double layer interaction or an entropic repulsion. ${ }^{36}$ Such entropic repulsion is known to depend on the size of the counter ion and when it is quite high, the vesicle surface interaction is weakened. This leads to non-rupture of the vesicles or coalescence of vesicles sitting on single bilayers.

Normally it takes minutes to hours for vesicles to rupture depending on the sizes. On the other hand, time needed to form SLBs can be in the range of minutes for the lipid concentrations employed here. If the rate of vesicle adsorption is faster compared to the rupture of individual vesicles, the surface density of intact vesicles can be higher. This indicates that rupture induced via critical vesicular coverage coexists with the rupture of isolated vesicles.

\section{Conclusions}

In conclusion, two types of topological structures form when DOPC vesicles are in contact with hydrophobic surface. (i) In the presence of low concentration of $\mathrm{NaCl}$ or ethanol, supported lipid bilayers are formed as with pure water. (ii) As concentration of $\mathrm{NaCl}$ is increased or when the size of the hydrated cation or anion changes ( as in $\mathrm{KCl}$ or $\mathrm{Na}_{2} \mathrm{SO}_{4}$ ) unruptured vesicles adsorb on initially spread bilayers and coalescence takes place leading to multibilayers. Thus, differences in degrees of hydration of DOPC in the presence of additives in the vesicles lead to different types of deformation behaviour leading to multibilayer formation. The vesicles in contact with solid surfaces need to be studied in more detail to understand the processes involved in adhesion, coalescence and spreading and no single experimental technique can give exhaustive information about the intermediate states.

\section{Acknowledgements}

The authors would like to thank the Department of Science and Technology (DST), Govt. of India for a project grant under which part of this work was carried out.

\section{References}

1. Plant A 1993 Langmuir 92764

2. Sackmann E 1996 Science 27143
3. Groves J T and Boxer S G 2002 Acc. Chem. Res. 35 149

4. Niemeyer C M and Mirkin C A 2004 Nanobiotechnology (Wiley)

5. Wacklin H P and Thomas R K 2007 Langmuir 23 7644

6. Dhathathreyan A and Steinem C 2008 Chem.Phys. Lett. 464226

7. Park J W and Lee G U 2006 Langmuir 115057

8. Rozovsky S, Kaizuka Y and Groves J T $2005 \mathrm{~J}$. Am. Chem. Soc. 12736

9. Lamberson E R, Cambrea L R and Hovis J S $2007 \mathrm{~J}$. Phys. Chem. B111 13664

10. Bene M, Billy D, Benda A, Speijer H, Hof M and Hermens W Th 2004 Langmuir 2010129

11. Claessens M M A E, Leermakers F A M, Hoekstra F A and Cohen Stuart M A 2007 Langmuir 236315

12. Salamon Z, Wang $Y$, Tollin $G$ and Macleod H A 1994 Biochim. Biophys. Acta 1195267

13. Axelrod D, Thompson N L and Burghardt T P 1983 J. Microsc. 12919

14. Reviakine I and Brisson A 2000 Langmuir 16 1806

15. Janshoff A, Galla H J and Steinem C 2000 Angew. Chem., Int. Ed. 394004

16. Benda A, Benes M, Mareček V, Lhotsky A, Hermens W Th and Hof M 2003 Langmuir 194120

17. Ge M and Freed J H 1998 Biophys. J. 74910

18. Bahng M K, Cho N J, Park J S and Kim K 1998 Langmuir 14463

19. Ha T H, Kim C H, Park J S and Kim K 2000 Langmuir 16871

20. Sauerbrey G 1959 Z.Phys. 155206

21. Muthuselvi L and Dhathathreyan A 2007 Biochim Biophys Acta 1774138

22. Muthuselvi L, Parthasarathi R and Dhathathreyan A 2006 Biochim. Biophys. Acta 17641767

23. Craig V S J and Plunkett M 2003 J. Collolid Interf. Sci. 262126

24. Edvardsson $M$, Svedhem S, Wang G, Richter R, Rodahl M and Kasemo B 2009 Anal. Chem. 81349

25. Roger-carmes T, Membrey F, Filiatre C and Foisy A 2002 J. Colloid Interface Sci. 245257

26. Lin Z and Ward M D 1996 Anal. Chem. 681285

27. Ha T H and Kim K, 2001 Langmuir 171999

28. Richter R P and Brisson A 2005 Biophys. J. 883422

29. Reviakine I and Brisson A 2000 Langmuir 161806

30. Radler J, Strey H and Sackmann E 1995 Langmuir 11 4539

31. Nollert P, Kiefer H and Jahnig F 1995 Biophys. J. 69 1447

32. Sandhya S, Muthuselvi L and Dhathathreyan A 2008 Appl. Surf. Sci. 2546575

33. Egawa H and Furusawa K 1999 Langmuir 151660

34. Bayerl T M and Bloom M 1990 Biophys. J. 58357

35. Cha T, Guo A and Zhu X Y 2006 Biophys. J. 901270

36. Israelachvili $\mathrm{J}$ and Wennerstrom H 1996 Nature 379 219 